\title{
Increased detection of polymicrobial septicaemia by repeat subculture
}

\author{
R. C. SPENCER AND CAROLINE D. NICOL
}

Department of Bacteriology, Royal Hallamshire Hospital, Glossop Road, Sheffield S10 2JF

\begin{abstract}
Summary. Repeated subcultures were performed on 572 blood cultures that had yielded single significant organisms and 55 from which contaminants had grown. This yielded 83 more isolates from 71 patients. Only one of the contaminated blood cultures yielded a further significant isolate. Additional antibiotic therapy was indicated in only six patients. Although repeated subculture increased the detection of additional isolates, it did not help in the management of most patients.
\end{abstract}

\section{Introduction}

The incidence of polymicrobial septicaemia (i.e. the isolation of more than one organism from a single blood culture) seems to be increasing and is associated with higher than expected case fatality rates (Saravolatz et al., 1978; Kiani et al., 1979; Kreger et al., 1980a; Ing et al., 1981). One way to improve the rate of polymicrobial isolations may be to reincubate positive blood cultures and then to repeat subcultures (Hansen and Hetmanski, 1983; Weinstein et al., 1983; Spencer et al., 1984) though the value of this approach has been disputed (Harkness et al., 1975; Stetz and Martin, 1985; Towne and Gay, 1985). In this prospective study we have analysed the results of repeated subculture of positive blood cultures over a 2-year period in an attempt to establish whether repeated subcultures increase the detection of polymicrobial isolates and are of benefit in the management of septicaemic patients.

\section{Materials and methods}

\section{Blood-culture media}

The following blood-culture media were used in 150 $\mathrm{ml}$ flat screw-capped bottles: (i) two bottles containing $100 \mathrm{ml}$ of Hartley Digest Broth (Oxoid) plus glucose $0.1 \%$ for aerobic culture; one bottle contained polyanethol sulphonate (Liquoid; Roche) $0.025 \%$; (ii) one bottle containing $100 \mathrm{ml}$ of Fastidious Anaerobe Broth (FAB; Lab M) for anaerobic culture. Each bottle was inoculated with $9 \mathrm{ml}$ of blood and incubated at $37^{\circ} \mathrm{C}$.

\section{Subculture technique}

Every bottle was visually inspected each day and any

Received 10 Sep. 1985; accepted 29 Oct. 1985. showing evidence of microbial growth, e.g., turbidity or haemolysis, were examined by Gram's stain and subculture. FAB bottles were routinely subcultured after $48 \mathrm{~h}$. All bottles were opened and sub-cultured after incubation for 7 days. Subcultures were performed with sterile disposable 10- $\mu$ l plastic loops (NUNC, Denmark). Onethird plates of Columbia (Oxoid) blood agar and lysedblood agar with kanamycin $70 \mathrm{mg} / \mathrm{L}$ and menadione $0.001 \%$ were inoculated at each sub-culture. The bloodagar cultures were incubated in air with $\mathrm{CO}_{2} 8 \%$ and the cultures on lysed-blood agar with kanamycin and menadione were incubated in anaerobic jars (Don Whitley Scientific Ltd.) in an atmosphere of $\mathrm{N}_{2} 80 \%, \mathrm{CO}_{2} 10 \%$ and $\mathrm{H}_{2} 10 \%$ (BOC Special Gases Ltd.). All plates were examined after incubation for 24 and $72 \mathrm{~h}$.

When any bottle yielded a potentially pathogenic organism or a contaminant, all three bottles were subcultured again after incubation for 3 and 10 or 14 days. On these occasions, repeat subcultures were performed with sterile disposable loops as before on to blood agar, lysed-blood agar with kanamycin and menadione, blood agar with nalidixic acid $10 \mathrm{mg} / \mathrm{L}$, and MacConkey's Agar (Oxoid). Incubation conditions were as previously described and all plates were examined after incubation for 24 and $72 \mathrm{~h}$.

\section{Identification of Isolates}

All isolates were identified by normal laboratory procedures (Cowan and Steel 1974; Pease, 1983).

\section{Results}

From a total of 7372 blood-culture sets studied over a 2-year period, $572(7.8 \%)$ yielded one clinically significant organism at the first subculture and 25 yielded more than one significant isolate. The 572 culture sets that yielded a single significant organism and 55 sets from which organisms regarded as contaminants were isolated were subcultured again after incubation for a further 3 and 
Table. Microbial species isolated after repeated subculture

\begin{tabular}{lccc} 
& \multicolumn{3}{c}{ Time of subculture (days) } \\
\cline { 2 - 4 } Organisms isolated & 3 only & 3 and 10 or 14 & 10 or 14 only \\
\hline Enterobacteriaceae & 0 & 25 & 13 \\
Non-fermenting gram- & 0 & 3 & 1 \\
$\quad$ negative bacilli & & & \\
Staphylococcus aureus & 0 & 4 & 0 \\
Staph. epidermidis & 0 & 4 & 0 \\
$\begin{array}{l}\beta \text {-haemolytic streptococci } \\
\text { Enterococci }\end{array}$ & 0 & 3 & 1 \\
x-haemolytic streptococci & 0 & 6 & 2 \\
Bacteroides fragilis & 0 & 5 & \\
Clostridium sp. & 1 & 4 & 2 \\
Other anaerobes & 0 & 4 & 0 \\
Candida alhicans & 0 & 1 & 0 \\
Total & 2 & 62 & 0
\end{tabular}

10 or 14 days. A further 83 isolates from 71 blood culture sets were obtained: the additional isolates were detected in $56\left(79^{\circ}\right)$ of these sets at the 3-day subculture. Details of these 83 organisms are shown in the table. Thirty-one patients were female and the overall mean age of patients was 55.1 years $(S D=19 \cdot 8)$. The additional organisms were also isolated from sites other than blood in $26(37 \%)$ patients-intravenous catheter tips 10; wounds, exudates etc. 9; urine 7. Only one of the initially contaminated blood-culture sets yielded an additional significant isolate. Of the 25 patients with polymicrobial septicaemia detected at the initial subculture, $14(56 \%)$ died, whereas of the 71 patients with polymicrobial detected at repeated subculture only $16(23 \%)$ died $\left(\chi^{2}=9.638: p<0.01\right)$. In an age- and sex-matched control group with comparable underlying clinical conditions, 18 $(25 \%)$ died.

\section{Discussion}

Incidences of polymicrobial septicaemia have been reported to be between 6 and $18 \%$ (Curtin et al., 1961; Hochstein et al.. 1965; Hermans and Washington, 1970) and there are indications that the isolation of more than one species of bacterium from patients with septicaemia is increasing (Saravolatz et al., 1978; Kiani et al., 1979). Because of its reported association with increased mortality (Hermans and Washington, 1970; Kiani et al., 1979), attempts have been made to increase the detection of polymicrobial septicaemia in recommending repeated subculture of previously positive blood cultures after incubation for a further 3 days (Hansen and Hetmanski, 1983) or 14 days (Weinstein et al., 1983). By so doing, Hansen and Hetmanski (1983) increased their polymicrobic bacteraemia rate from $9.1 \%$ to $27 \%$ of clinically significant patient episodes. This had a direct impact on patient management. Weinstein et al. (1983) reported a rate of $18.4 \%$ and offered the repeat subculturing of previously positive cultures as one possible explanation of this high figure. This approach has been discounted by other workers (Stetz and Martin. 1985; Towne and Gay, 1985) who found that repeated subculturing after 3 days did not increase the incidence, added significantly to the time and cost of evaluating blood cultures, and did not improve overall patient care. Our study has shown an increase in incidence from $25(4.2 \%$ of total positive blood cultures) to $96(16 \%)$, with the majority $(79 \%)$ found at the 3-day subculture when it would be of most help in patient management. Only $21 \%$ were found at the 10 - or 14-days subculture recommended by Weinstein et al. (1983). Hansen and Hetmanski (1983) used a radiometric system (BACTEC, Johnson Laboratories) as did Towne and Gay (1985). Weinstein et al. (1983) sampled $15 \mathrm{ml}$ of blood in a broth medium whereas Stetz and Martin (1985) sampled $20 \mathrm{ml}$ of blood and we obtained c. $30 \mathrm{ml}$ of blood from our patients. It has been shown that there is a direct relationship between the volume of blood sampled and the yield of positive cultures (Ilstrup and Washington, 1983). Thus the smaller inoculum of blood $(7-10 \mathrm{ml})$ used in the BACTEC system may increase the time necessary to detect microbial growth by subculture and, therefore, re-culturing after $72 \mathrm{~h}$ could provide the time required to detect those organisms present which were not isolated at an earlier time. The patients studied by Weinstein et al. (1983) included a large proportion of immunocompromised patients which could explain the high incidence they report. This phenomenon has also been reported by others (Hermans and Washington, 1970; Kiani et al. 1979) and in our study the patients in whom a subsequent organism was found predominantly suffered from haematological malignancies, carcinoma, or hepatic or renal disorders, $37(52 \%)$ patients being on immunosuppressive or cancer chemotherapy. Invasive procedures also appeared to be important; $34(48 \%)$ of patients had an intravenous or urinary catheter in situ. Inappropriate antibiotic therapy is associated with an increased incidence of bacteraemic shock and death 
(Kreger et al., 1980b) but because most of our patients were suffering from serious underlying disease, most of them were receiving more than one antibiotic. As a result additional antibiotics were only considered necessary in $6(8.5 \%)$ of these later diagnosed patients.

Is the increase in polymicrobial isolates of significance to the patient? We found a significantly increased mortality $(56 \%)$ in those patients with primary polymicrobial septicaemia, but no increase in those patients in whom additional isolates were found subsequently when compared with an ageand sex-matched control group with comparable underlying conditions whose blood cultures yielded only single bacterial species $(23 \%$ and $25 \%$ respectively). This finding was similar to that of Kiani et al. (1979). Thus, although repeat subcultures increased

\section{REFERENCES}

Cowan S T 1974 Cowan and Steel's manual for the identification of medical bacteria, 2nd edn. Cambridge University Press, Cambridge.

Curtin J A, Petersdorf R G, Bennett I L 1961 Pseudomonas Bacteremia: A Review of 91 cases. Annals of Internal Medicine 54:1077-1107.

Hansen S L, Hetmanski J 1983 Enhanced detection of polymicrobic bacteremia by repeat sub culture of previously positive blood cultures. Journal of Clinical Microbiology 18:208-210.

Harkness J L, Hall M, Ilstrup D M, Washington J A 1975 Effects of atmosphere of incubation and of routine subcultures on detection of bacteremia in vacuum blood culture bottles. Journal of Clinical Microbiology 2:296-299.

Hermans P E, Washington J A 1970 Polymicrobial bacteremia. Annals of Internal Medicine 73:387-392.

Hochstein H D. Kirkham W R, Young V M 1965 Recovery of more than 1 organism in septicemias. New England Journal of Medicine 273:468-474.

Ilstrup D M, Washington J A 1983 The importance of volume of blood cultured in the detection of bacteremia and fungemia. Diagnostic Microbiology and Infectious Diseases 1:107-110.

Ing A F M, McLean P H, Meakins J L 1981 Multiple organism bacteremia in the surgical intensive care unit: a sign of intraperitoneal sepsis. Surgery 90:779-786.

Kiani D, Quinn E L, Burch K H, Madhavan T, Saravolatz L D, Neblett T R 1979 The increasing importance of polymicrobial bacteremia. Journal of the American Medical Association 242:1044-1047.

Kreger B E, Craven D E, Carling P C, McCabe W R 1980a Gram-negative bacteremia. III. Reassessment of etiology, epidemiology and ecology in 612 patients. American Journal of Medicine 68:332-343. the detection of additional isolates, it did not help in the management of most patients. In these patients, the additional organisms seem to be present originally, but in too low numbers to be of consequence. This is reflected in the significantly lower mortality rate. Only one of the contaminated blood-culture sets yielded an additional significant isolate. As in a previous report (Spencer and Nicol, 1985) we found that repeating subcultures after incubation for 3 days increased polymicrobial isolation, but that such a procedure, whilst increasing the time and cost of processing blood culture samples, did not benefit the vast majority of patients.

This work was supported by a National Health Service Local Research Grant.

Kreger B E, Craven D E, McCabe W R 1980b Gram-negative bacteremia. IV. Re-evaluation of clinical features and treatment in 612 patients. American Journal of Medicine 68:344-355.

Pease A A 1983 Biochemical identification of the enterobacteriaceae using a multipoint inoculation system. Medical Laboratory Sciences 40:349-353.

Saravolatz L D, Burch K H, Quinn E L, Cox F, Madhavan T, Fisher E 1978 Polymicrobial infective endocarditis: an increasing clinical entity. American Heart Journal 95:163168.

Spencer R C, Courtney S P, Nicol C D 1984 Polymicrobial septicaemia due to Clostridium difficile and Bacteroides fragilis. British Medical Journal 289:531-532.

Spencer R C, Nicol C D 1985 Polymicrobial septicaemia. Lancet 1:1210.

Stetz E M, Martin W J 1985 Repeat subculture of known positive blood cultures: costly and ineffective in detecting polymicrobial bacteremias. Diagnostic Microbiology and Infectious Diseases 3:113-118.

Towne A R, Gay R M 1985 Evaluation of the efficacy of reincubation and subsequent subculture of initially positive blood cultures in the detection of additional clinically significant isolates. Journal of Clinical Microbiology 21:155157.

Weinstein M P, Reller L B Murphy J R, Lichtenstein K A 1983 The clinical significance of positive blood cultures: a comprehensive analysis of 500 episodes of bacteremia and fungemia in adults. I. Laboratory and epidemiologic observations. Review of Infectious Diseases 5:35-53. 